\section{The White Cerebellum Sign: An Under-recognized Red Flag Finding with Grave Prognosis}

Sir,

Road traffic accidents are a major cause of death in middle and low income countries. ${ }^{1}$ While some of these deaths may be caused from the direct trauma at the site of accident, a significant proportion of these deaths occur later due to traumatic brain injury, management of which remains time critical. This can be difficult due to non-availability of neurosurgical specialists in smaller centres compounded by a lack of pre-hospital care and organised land and air ambulance facilities to shift the victims from the site of accident to a specialist trauma centre. 1

A CT scan of the brain remains the investigation of choice in suspected traumatic brain injuries. It is important that the reporting radiologist remains aware of the specific findings that may be identified which may indicate a poor prognosis after traumatic brain injuries, as subsequent management decisions may be taken depending on that findings. One such red flag sign is the white cerebellum or the reversal sign identified on CT scan imaging.

A 44-year-old man was transferred to a neurosurgical centre in north-eastern India three days after being involved in a road traffic accident. He was symptomatically managed initially in a non-specialist hospital in the intensive care unit. As his condition gradually deteriorated with worsening of GCS, he was intubated and ventilated and a decision to transfer to the neurosurgical centre was agreed with the family. He was deeply comatose at admission.

CT scan of the brain done at admission showed temporal contusions with mass effect with doubtful white cerebellum sign (Figure 1). Family was counselled regarding extreme poor prognosis. However, they insisted on neurosurgical intervention, which was performed the same evening. The contusions were evacuated and decompression was achieved. However, postoperative CT scan showed profound white cerebellum sign (Figure 2). The patient remained ventilated with GCS fluctuating between 3 to 4 over the next 72 hours. Following subsequent discussions and counselling, it was agreed with the family that the chances of survival were absent, so the family decided to transfer the patient back to their local hospital. The patient died on day 10 after the accident.

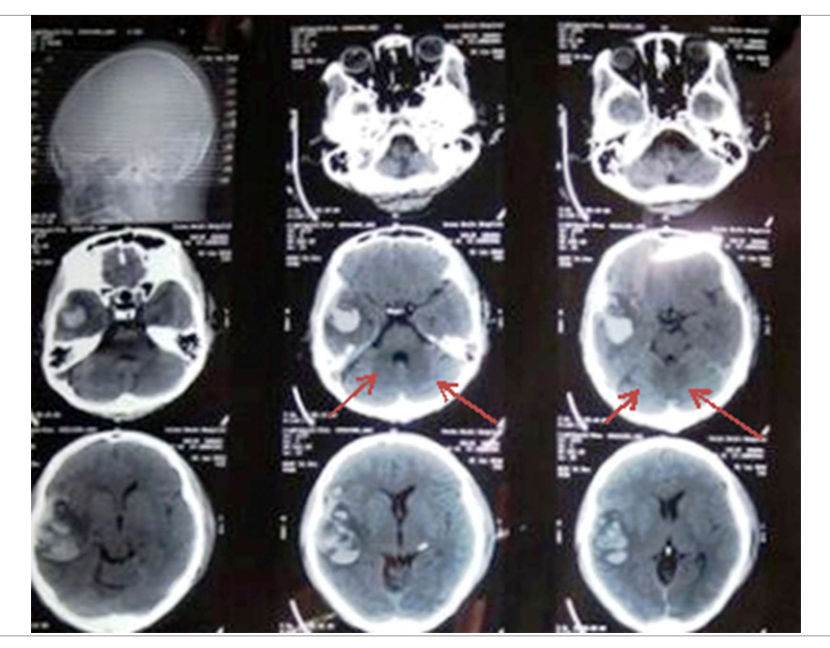

Figure 1: Preoperative CT shows possible white cerebellum sign (shown by arrows).

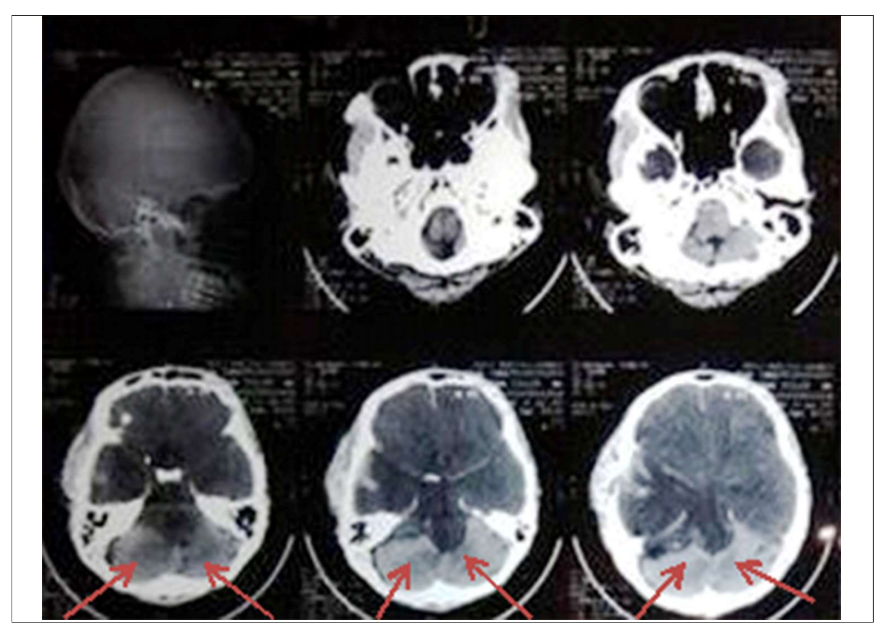

Figure 2: Postoperative CT shows profound white cerebellum sign (shown by arrows).

The white cerebellum sign is considered to result from diffuse cerebral oedema leading to loss of normal differentiation of grey-white matter of the cerebral parenchyma. It is postulated that in severe anoxia there is a preferential blood flow to the posterior fossa structures which preserves the normal appearance of the cerebellum; hence, it looks brighter on CT evaluation. ${ }^{2}$ These density changes seen on CT scan images are known to progress to complete reversal of the cortical grey-white matter densities with relative preservation of normal to high density of the central structures and cerebellum and is often referred to as the white cerebellum or reversal sign. ${ }^{3}$

The white cerebellum sign is suggestive of severe hypoxic ischaemic brain injury. It has been described in a number of conditions, e.g. birth asphyxia, head injury, abusive head trauma, drowning, status epilepticus, hypothermia, meningoencephalitis, postpartum seizures and other causes of global hypoperfusion.2,4 It is 
important that the radiologists reporting the CT scan, following a brain injury actively look for this sign as it may not be obvious in some cases and has got grave consequences if therapeutic interventions such as lumbar puncture subsequently gets performed which may result in downward herniation as a result of a pressure gradient between the supratentorial and infratentorial compartment. 5 Detection of this sign is usually associated with poor prognosis and irreversible brain injury and, therefore, is important from a diagnostic, therapeutic, and prognostic decision making process for the clinicians.

\section{CONFLICT OF INTEREST:}

Authors declared no conflict of interest.

\section{AUTHORS' CONTRIBUTION:}

SA: Concept, manuscript editing, and provided expert neurosurgical opinion.

TB: Data collection, case study summarization, obtaining electronic images, literature search.

SPP: Manuscript preparation and revision; literature search, submission and correspondence.

\section{REFERENCES}

1. Oestern HJ, Garg B, Kotwal P. Trauma care in India and Germany. Clin Orthop Relat Res 2013; 471:2869-77.

2. Singh $D$, Sharma $A$. White cerebellum sign: Red flag in the emergency department. CJEM 2014; 16:413.

3. Maciel CB, D'Amico RS, Gupta A. The reversal sign: An ominous imaging finding. Neurohospitalist 2015; 5:251-2.

4. Krishnan P, Chowdhury SR. "White cerebellum" sign - A dark prognosticator. J Neurosci Rural Pract 2014; 5:433.

5. Chalela JA, Rothlisberger J, West B, Hays A. The white cerebellum sign: An under recognized sign of increased intracranial pressure. Neurocrit Care 2013; 18:398-9.

Shameem Ahmed ${ }^{1}$, Taposh Biswas ${ }^{1}$ and Siba Prosad Paul

1 Department of Neurosurgery, Apollo Hospitals, (Unit: International Hospital), Guwahati, India

2 Department of Paediatrics, Torbay Hospital, Torquay, UK Correspondence to: Dr. Siba Prosad Paul, Department of Paediatrics, Torbay Hospital, Vowden Hall, Lawes Bridge, Torquay, TQ2 7AA, UK

E-mail: siba@doctors.org.uk

Received: February 22, 2019; Revised: March 26, 2019; Accepted: March 26, 2019 